\title{
Synthesis of structural elements of the capsular polysaccha- rides of Streptococcus pneumoniae types 6A and 6B
}

Ted M. Slaghek, Anita H. van Oijen, Augustinus A. M. Maas, Johannis P. Kamerling, and Johannes F. G. Vliegenthart

Department of Bio-Organic Chemistry, Utrecht University, Transitorium III, P.O. Box 80.075, NL-3508 TB Utrecht (The Netherlands)

(Received June 17th, 1989; accepted for publication, February 20th, 1990)

\section{ABSTRACT}

$O$ - $\alpha$-D-Glucopyranosyl-( $1 \rightarrow 3$ )- $\alpha, \beta$-L-rhamnopyranose (15), $O$ - $\alpha$-D-galactopyranosyl-(1 $\rightarrow 3$ )- $O$ - $\alpha$-Dglucopyranosyl-( $1 \rightarrow 3)-\alpha, \beta$-L-rhamnopyranose $(17), O-\alpha$-D-galactopyranosyl-(1 $\rightarrow 3)-O$ - $\alpha$-D-glucopyrano-

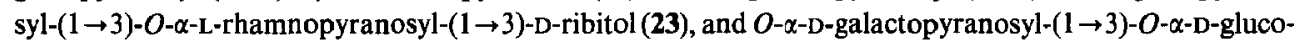
pyranosyl-( $1 \rightarrow 3$ )- $O-\alpha$-L-rhamnopyranosyl-( $1 \rightarrow 4)$-D-ribitol $(27)$, which are structural elements of the capsular polysaccharides of Streptococcus pneumoniae types 6A and 6B $\{[\rightarrow 2)-\alpha-D-G a l p-(1 \rightarrow 3)-\alpha-D-G l c p-(1 \rightarrow 3)-$ $\alpha$-L-Rhap- $(1 \rightarrow \mathrm{X})$-D-Rib-ol- $\left.(5-\mathrm{P} \rightarrow]_{n} ; 6 \mathrm{~A} \mathrm{X}=3,6 \mathrm{~B} \mathrm{X}=4\right\}$, have been synthesised. Ethyl 3-O-allyl-2,4,6-tri$O$-benzyl-1-thio- $\beta$-D-glucopyranoside (3) was coupled with benzyl 2,4-di- $O$-benzyl- $\alpha$-L-rhamnopyranoside (4), and subsequent deallylation $(\rightarrow 14)$ and debenzylation gave 15. Condensation of 14 with ethyl $2,3,4,6-$ tetra-O-benzyl-1-thio- $\beta$-D-galactopyranoside (2) followed by debenzylation gave 17. Acetylation of 17 followed by removal of AcO-1, conversion into the imidate, coupling with 1,2,4,5-tetra- $O$-benzyl-D-ribitol (11), deacetylation, and debenzylation gave 23. Coupling of the imidate with 1- $O$-allyloxycarbonyl-2,3,5-tri$O$-benzyl-D-ribitol (12) followed by deallyloxycarbonylation, deacetylation, and debenzylation yielded 27.

\section{INTRODUCTION}

As part of our studies on the development of synthetic vaccines, based on oligosaccharide conjugates, against infections by Streptococcus pneumoniae serotypes, we have described the synthesis of an essential building block for the preparation of higher oligomers corresponding with fragments of the capsular polysaccharide of types 6A and 6B, namely, 4-methoxybenzyl 2,4-di- $O$-benzyl-3-O-[2,4,6-tri- $O$-benzyl-3-O(3,4,6-tri- $O$-benzyl- $\alpha$-D-galactopyranosyl)- $\alpha$-D-glucopyranosyl]- $\alpha$-L-rhamnopyranoside'.

We now report the synthesis of the repeating units of the capsular polysaccharides of the serotypes $6 \mathrm{~A}$ and 6B, namely, $\alpha-\mathrm{D}-\mathrm{Gal} p-(1 \rightarrow 3)-\alpha-\mathrm{D}-\mathrm{Gl} p-(1 \rightarrow 3)-\alpha-\mathrm{L}-\mathrm{Rh}$ ha $(1 \rightarrow 3)$-D-Rib-ol (23) and $\alpha$-D-Gal $p$ - $(1 \rightarrow 3)-\alpha$-D-Glcp-(1 $\rightarrow 3)-\alpha-\mathrm{L}-\mathrm{Rha} p-(1 \rightarrow 4)$-D-Rib-ol (27), respectively, together with the structural elements $\alpha-\mathrm{D}-\mathrm{Glc} p-(1 \rightarrow 3)-\alpha, \beta-\mathrm{L}-\mathrm{Rh} p$ (15) and $\alpha-\mathrm{D}-\mathrm{Gal} p-(1 \rightarrow 3)-\alpha-\mathrm{D}-\mathrm{Gl} p-(1 \rightarrow 3)-\alpha, \beta$-L-Rhap (17).

\footnotetext{
"Author for correspondence.

0008-6215/90/\$03.50 C 1990 - Elsevier Science Publishers B.V.
} 


\section{RESULTS AND DISCUSSION}

For the synthesis of 23 and 27, the three monosaccharide synthons ethyl 3-Oallyl-2,4,6-tri- $O$-benzyl-1-thio- $\beta$-D-glucopyranoside ${ }^{1}$ (3), benzyl 2,4 -di- $O$-benzyl- $\alpha$-Lrhamnopyranoside $^{2}(4)$, and 1-O-allyloxycarbonyl-2,3,5-tri- $O$-benzyl-D-ribitol ${ }^{3}$ (12) were synthesised as described earlier, and the two monosaccharide synthons 2 for D-Gal and 11 for D-Rib-ol were prepared as follows.

Ethyl 1-thio- $\beta$-D-galactopyranoside ${ }^{4-6}$ (1) was benzylated to yield ethyl $2,3,4,6$ tetra- $O$-benzyl-1-thio- $\beta$-D-galactopyranoside $(2,81 \%)$.

Methyl 5- $O$-benzyl- $\alpha, \beta$-D-ribofuranoside ${ }^{7}(5)$ was selectively benzylated at $\mathrm{C}-2$, using a phase-transfer catalyst ${ }^{1,2,8}(\rightarrow 6,44 \%)$. Subsequent allylation $(\rightarrow 7,93 \%)$, demethylation with $1.2 \mathrm{M}$ hydrochloric acid in 1,4-dioxane $(\rightarrow 8,79 \%)$, reduction with sodium borohydride in ethanol $(\rightarrow 9,85 \%)$, benzylation $(\rightarrow 10,85 \%)$, deallylation with $\mathrm{KO}^{t} \mathrm{Bu}$ in $N, N$-dimethylformamide, and acid hydrolysis gave 1,2,4,5-tetra- $O$-benzyl-Dribitol $(11,71 \%)$.

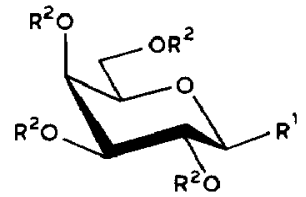

$1 R^{1}=S E t, R^{2}=H$

$2 R^{\prime}=S E t, R^{2}=B n$

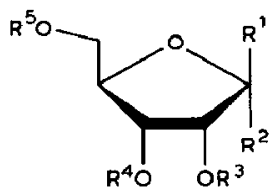

$3 R^{1}, R^{2}=H, O M e, R^{3}=R^{4}=H, R^{5}=B n$

$6 R^{1}=$ OMe, $R^{2}=R^{4}=H, R^{3}=R^{5}=$ Bn

$7 R^{1}=$ OMe, $R^{2}=H, R^{3}=R^{5}=B n, R^{4}=$ allyl

B $R^{\prime}, R^{2}=H, O H, R^{3}=R^{5}=B n, R^{4}=$ al|y|

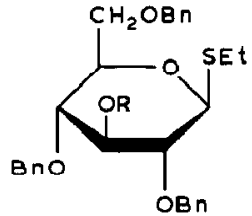

$3 \mathrm{R}=\mathrm{a}|| \mathrm{yl}$

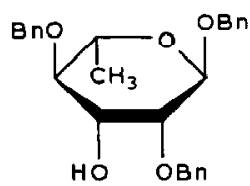

4<smiles>OC[C@@H]([18OH])[C@H](O)[C@H]([18OH])C[18O]</smiles>

9 $R^{1}=R^{4}=H, R^{2}=R^{5}=B n, R^{3}=$ aly $\mid$

$10 R^{1}=R^{2}=R^{4}=R^{5}=B n, R^{3}=$ ol|y|

$11 R^{\prime}=R^{2}=R^{4}=R^{5}=B n, R^{3}=H$

$12 R^{4}=A O C, R^{2}=R^{3}=R^{5}=B \cap, R^{4}=H$

$(A O C=$ allyloxycarbony 1$)$

Coupling of ethyl 3- $O$-allyl-2,4,6-tri- $O$-benzyl-1-thio- $\beta$-D-glucopyranoside ${ }^{1}$ (3) and benzyl 2,4-di- $O$-benzyl- $\alpha$-L-rhamnopyranoside ${ }^{2}$ (4) in ether, using methyl triflate ${ }^{9}$ as the promoter, gave $13(61 \%)$. The allyl group of 13 was removed using $\mathrm{KO}^{\mathrm{t}} \mathrm{Bu}$ in $N, N$-dimethylformamide followed by acid hydrolysis to yield $14(93 \%)$. Catalytic hydrogenolysis of 14 over $\mathrm{Pd}-\mathrm{C}$ removed the benzyl groups and gave disaccharide 15 $(80 \%)$. Condensation of 14 with the Gal synthon 2 in ether, using methyl triflate ${ }^{9}$ as the promoter, afforded $16(57 \%)$. After catalytic hydrogenolysis of $16(\rightarrow 17,89 \%)$ and acetylation in pyridine/acetic anhydride in the presence of a trace of dimethylaminopyridine at $50^{\circ}(\rightarrow 18)$, AcO-1 was removed with hydrazine acetate in $N, N$-dimethylforma- 
mide $^{10}(\rightarrow 19,88 \%)$. Subsequent imidation using trichloroacetonitrile and 1,8-diazabicyclo[5.4.0]undec-7-ene (DBU) as a base ${ }^{11}$ afforded 2,4-di- $O$-acetyl-3-O-[2,4,6-tri-Oacetyl-3-O-(2,3,4,6-tetra- $O$-acetyl- $\alpha$-D-galactopyranosyl)- $\alpha$-D-glucopyranosyl]- $\alpha$-Lrhamnopyranosyl trichloroacetimidate $(20,81 \%)$.

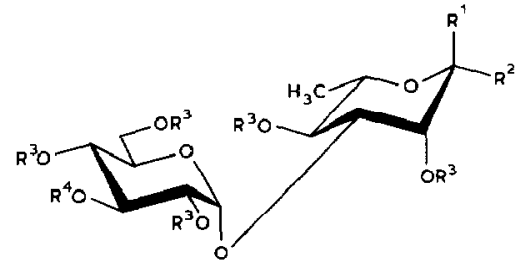

$13 R^{1}=O B n, R^{2}=H, R^{3}=B n, R^{4}=a l|y|$ $14 R^{4}=O B n, R^{2}=R^{4}=H, R^{3}=B n$ $15 R^{9}, R^{2}=H, O H, R^{3}=R^{4}=H$

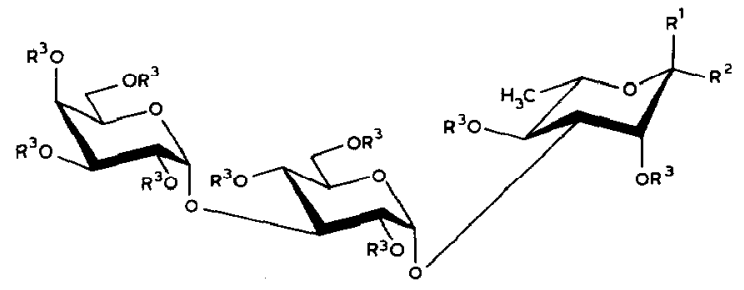

$16 R^{1}=O B n, R^{2}=H, R^{3}=B n$

$17 R^{1}, R^{2}=H, O H, R^{3}=H$

$18 R^{1}, R^{2}=H, O A C, R^{3}=A C$

$19 R^{\prime}=O H, R^{2}=H, R^{3}=A C$

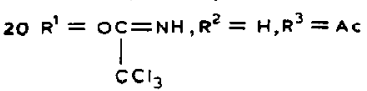

Coupling of 20 with the Rib-ol synthon 11 in dichloromethane, using trimethylsilyl triflate as the promoter, gave $21(90 \%)$, which was saponified $(\rightarrow 22)$ and debenzylated to afford $23(93 \%)$. In a similar way, 20 was condensed with the Rib-ol synthon 12 (ref. 3) to yield $\mathbf{2 4}(91 \%)$ ). Subsequent removal of the allyloxycarbonyl group with palladium tetrakis[triphenylphosphine] $]^{3}(\rightarrow 25,82 \%)$, deacetylation $(\rightarrow 26)$, and catalytic hydrogenolysis gave $27(90 \%)$. The ${ }^{1} \mathrm{H}$ - and ${ }^{13} \mathrm{C}-\mathrm{n}$.m.r. data of the tetrasaccharidealditol 27 were identical to those of the tetrasaccharide-alditol isolated ${ }^{12}$ from the capsular polysaccharide $6 \mathrm{~B}$ by hydrolysis with $\mathrm{HF}$.

The di- and tri-saccharides $15,17,23$, and 27 are being tested in immunological inhibition experiments in order to evaluate the precise antigenic determinant of $6 \mathrm{~A}$ and 6B.

EXPERIMENTAL

General methods. - ' 'H-N.m.r. spectra $(60,360$, and $500 \mathrm{MHz})$ were recorded at $25^{\circ}$ with a Varian 360 , Bruker HX 360 , or Bruker AM 500 spectrometer. The ${ }^{13}$ C-n.m.r. spectra $(50 \mathrm{MHz})$ were recorded at $25^{\circ}$ with a Bruker WP-200 spectrometer. Chemical shifts $(\delta)$ are given in p.p.m. relative to internal $\mathrm{Me}_{4} \mathrm{Si}\left(\mathrm{CDCl}_{3}\right)$ or internal sodium 4,4-dimethyl-4-silapentane-1-sulfonate $\left(\mathrm{D}_{2} \mathrm{O}\right.$; indirectly to internal acetone, $\left.\delta 2.225\right)$ for ${ }^{1} \mathrm{H}$, and to internal $\mathrm{Me}_{4} \mathrm{Si}\left(\mathrm{CDCl}_{3}\right.$; indirectly to $\left.\mathrm{CDCl}_{3}, \delta 76.9\right)$ and external $\mathrm{Me}_{4} \mathrm{Si}\left(\mathrm{D}_{2} \mathrm{O}\right.$; indirectly to internal acetone, $\delta 31.55$ ) for ${ }^{13} \mathrm{C}$ data. 

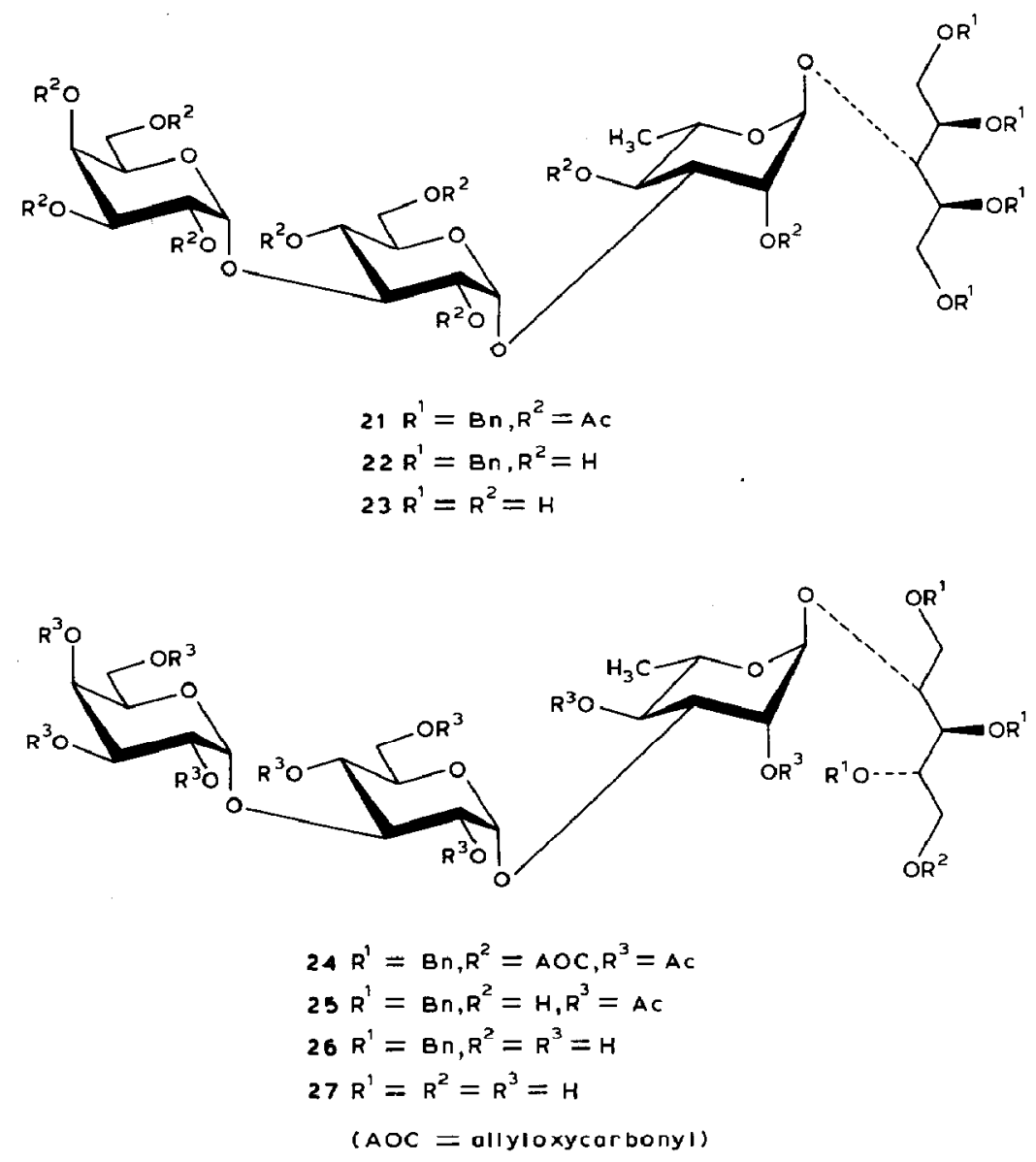

Column chromatography was performed on Kieselgel 60 (Merck, $<230$ mesh) and fractions were monitored by t.l.c. on Kieselgel $60 \mathrm{~F}_{254}$ (Merck). Detection was effected by charring with sulfuric acid after examination under u.v. light. Optical rotations were measured at $20^{\circ}$ with a Perkin-Elmer 241 polarimeter, using a $10-\mathrm{cm}$ microcell. Evaporations were conducted in vacuo at $40^{\circ}$ (bath). All solvents were distilled from appropriate drying agents.

Ethyl 2,3,4,6-tetra-O-henzyl-1-thio- $\beta$-D-galactopyranoside (2). - To a suspension of sodium hydride (6 g, 8 equiv.) in $N, N$-dimethylformamide $(50 \mathrm{~mL})$ was added dropwise at $0^{\circ}$ a solution of ethyl 1-thio- $\beta$-D-galactopyranoside ${ }^{6}(1 ; 3.84 \mathrm{~g}, 17.1 \mathrm{mmol})$ and benzyl bromide $(12.2 \mathrm{~mL})$ in $N, N$-dimethylformamide $(50 \mathrm{~mL})$. The mixture was stirred for $2 \mathrm{~h}$ at room temperature $\left[R_{\mathrm{F}} 0.80,9: 1\right.$ light petroleum (b.p. $40-60^{\circ}$ )-ethyl acetate], the excess of sodium hydride was destroyed with methanol, the mixture was poured onto crushed ice and extracted with ether $(3 \times 50 \mathrm{~mL})$, and the combined extracts were dried $\left(\mathrm{MgSO}_{4}\right)$, filtered, and concentrated. The residue was purified by column chromatography [9:1 light petroleum (b.p. $40-60^{\circ}$ )-ethyl acetate] to yield 2, isolated as a syrup $(8.10 \mathrm{~g}, 81 \%),[\alpha]_{\mathrm{D}}-4^{\circ}\left(c 1\right.$, chloroform). N.m.r. data $\left(\mathrm{CDCl}_{3}\right){ }^{1} \mathrm{H}, \delta$ 
$1.290\left(\mathrm{t}, 3 \mathrm{H}, J_{\mathrm{CH}_{3}, \mathrm{CH}_{2}} 7.5 \mathrm{~Hz}, \mathrm{SCH}_{2} \mathrm{CH}_{3}\right), 2.729\left(\mathrm{~m}, 2 \mathrm{H}, \mathrm{SCH}_{2} \mathrm{CH}_{3}\right), 3.821\left(\mathrm{t}, 1 \mathrm{H}, J_{1,2}=\right.$ $\left.J_{2.3}=9.6 \mathrm{~Hz}, \mathrm{H}-2\right), 3.951$ (bd, $\left.1 \mathrm{H}, J_{3,4} 2.8 \mathrm{~Hz}, \mathrm{H}-4\right), 4.428$ (d, $\left.1 \mathrm{H}, \mathrm{H}-1\right), 4.399$ (d), 4.475 (d), 4.609 (d), 4.723 (s), 4.788 (d), 4.875 (d), and 4.946 (d) ( $\left.8 \mathrm{H}, 4 \mathrm{PhCH}_{2} \mathrm{O}\right), 7.250-7.396$ (m, $20 \mathrm{H}, 4 \mathrm{Ph}) ;{ }^{13} \mathrm{C}, \delta 14.9\left(\mathrm{SCH}_{2} \mathrm{CH}_{3}\right), 24.6\left(\mathrm{SCH}_{2} \mathrm{CH}_{3}\right), 68.6,72.5,73.4,74.2$, and 75.6 (C-6 and $\left.4 \mathrm{C}_{6} \mathrm{H}_{5} \mathrm{CH}_{2}\right), 73.5,77.0,78.3,83.9$, and $85.1(\mathrm{C}-1,2,3,4,5), 127.3-128.2$ and 137.1-138.1 $\left(\mathrm{C}_{6} \mathrm{H}_{5} \mathrm{CH}_{2}\right)$.

Anal. Calc. for $\mathrm{C}_{36} \mathrm{H}_{40} \mathrm{O}_{5} \mathrm{~S}: \mathrm{C}, 73.93 ; \mathrm{H}, 6.91$. Found: C, 73.80; H, 6.85 .

Methyl 2,5-di-O-benzyl- $\beta$-D-ribofuranoside (6). - To a solution of methyl 5-Obenzyl- $\alpha, \beta$-D-ribofuranoside ${ }^{7}(5 ; 1.25 \mathrm{~g}, 4.92 \mathrm{mmol})$ in dichloromethane $(49.3 \mathrm{~mL})$ were added tetrabutylammonium bromide $(396 \mathrm{mg})$, benzyl bromide $(6.45 \mathrm{~mL})$, and aqueous $10 \%$ sodium hydroxide $(4.93 \mathrm{~mL})$. After stirring overnight, the mixture $\left(R_{\mathrm{F}} 0.36,5: 1\right.$ toluene-ethyl acetate) was washed with water $(30 \mathrm{~mL})$, dried $\left(\mathrm{MgSO}_{4}\right)$, filtered, and concentrated. The residue was purified by column chromatography (5:1 toluene-ethyl acetate) to yield 6, isolated as a syrup ( $750 \mathrm{mg}, 44 \%),[\alpha]_{\mathrm{D}}+4^{\circ}(c 1$, dichloromethane). N.m.r. data $\left(\mathrm{CDCl}_{3}\right):{ }^{1} \mathrm{H}, \delta 3.331$ (s, $3 \mathrm{H}, \mathrm{OMe}$ ), 3.554 (dd, $1 \mathrm{H}, J_{5 \mathrm{a}, 5 \mathrm{~b}} 10.4 \mathrm{~Hz}, \mathrm{H}-5 \mathrm{~b}$ ), 3.657 (dd, $1 \mathrm{H}, \mathrm{H}-5 \mathrm{a}), 3.859$ (dd, $\left.1 \mathrm{H}, J_{2,3} 5.0, J_{1.2} 1.2 \mathrm{~Hz}, \mathrm{H}-2\right), 4.097$ (m, $1 \mathrm{H}, J_{4,5 \mathrm{a}} 3.7, J_{4,5 b}$ $6.2 \mathrm{~Hz}, \mathrm{H}-4), 4.161$ (m, $1 \mathrm{H}, \mathrm{H}-3), 4.596$ (s), 4.622 (d), and 4.733 (d) (4 H, $2 \mathrm{PhCH}_{2} \mathrm{O}$ ), $4.905(\mathrm{~d}, 1 \mathrm{H}, \mathrm{H}-1), 7.265-7.368(\mathrm{~m}, 10 \mathrm{H}, 2 \mathrm{Ph}) ;{ }^{13} \mathrm{C}, \delta 54.5$ (OMe), $71.2(\mathrm{C}-3), 71.1,72.1$, and $72.7\left(2 \mathrm{C}_{6} \mathrm{H}_{5} \mathrm{CH}_{2}\right.$ and $\left.\mathrm{C}-5\right), 81.4$ and $82.5(\mathrm{C}-2,4), 105.3(\mathrm{C}-1), 127.0-128.0,136.8$, and $137.8\left(\mathrm{C}_{6} \mathrm{H}_{5} \mathrm{CH}_{2}\right)$.

Anal. Calc. for $\mathrm{C}_{20} \mathrm{H}_{24} \mathrm{O}_{5}: \mathrm{C}, 69.74 ; \mathrm{H}, 7.04$. Found: $\mathrm{C}, 69.66 ; \mathrm{H}, 7.24$.

Methyl 3-O-allyl-2,5-di-O-benzyl- $\beta$-D-ribofuranoside (7). - A solution of 6 (780 $\mathrm{mg}, 2.27 \mathrm{mmol})$ and allyl bromide $(0.3 \mathrm{~mL}, 1.5$ equiv.) in $N, N$-dimethylformamide ( 5 $\mathrm{mL}$ ) was added dropwise to a suspension of sodium hydride (110 $\mathrm{mg}, 2$ equiv.) in $N, N$-dimethylformamide $(5 \mathrm{~mL})$ at $0^{\circ}$. The mixture was stirred for $2 \mathrm{~h}$ at room temperature $\left(R_{\mathrm{F}} 0.16,95: 5\right.$ toluene-acetone), the excess of sodium hydride was destroyed with methanol, the mixture was poured onto crushed ice and extracted with ether $(3 \times 20 \mathrm{~mL})$, and the combined extracts were dried $\left(\mathrm{MgSO}_{4}\right)$, filtered, and concentrated. The residue was purified by column chromatography (95:5 tolueneacetone) to yicld 7 , isolated as a syrup $(814 \mathrm{mg}, 93 \%),[\alpha]_{\mathrm{D}}+25^{\circ}(\mathrm{c} 1$, dichloromethane). N.m.r. data $\left(\mathrm{CDCl}_{3}\right)$ : ${ }^{~} \mathrm{H}, \delta 3.316(\mathrm{~s}, 3 \mathrm{H}, \mathrm{OMe}), 3.547\left(\mathrm{dd}, 1 \mathrm{H}, J_{4,5 \mathrm{~b}} 5.8, J_{5 \mathrm{a}, 5 \mathrm{~b}} 10.6 \mathrm{~Hz}\right.$, $\mathrm{H}-5 \mathrm{~b}$ ), 3.646 (dd, $\left.1 \mathrm{H}, J_{4.5 \mathrm{sa}} 3.7 \mathrm{~Hz}, \mathrm{H}-5 \mathrm{a}\right), 3.853$ (dd, $1 \mathrm{H}, J_{2.3} 4.6, J_{1.2} 0.8 \mathrm{~Hz}, \mathrm{H}-2$ ), 3.904-4.026 (m, $3 \mathrm{H}, \mathrm{OCH}_{2} \mathrm{CH}=\mathrm{CH}_{2}$ and $\left.\mathrm{H}-3\right), 4.230(\mathrm{~m}, 1 \mathrm{H}, \mathrm{H}-4), 4.561,4.605,4.642$, and $4.688\left(4 \mathrm{~d}, 4 \mathrm{H}, 2 \mathrm{PhCH}_{2} \mathrm{O}\right), 4.905(\mathrm{bs}, 1 \mathrm{H}, \mathrm{H}-1), 5.143$ and $5.223(2 \mathrm{~m}, 2 \mathrm{H}$, $\left.\mathrm{OCH}_{2} \mathrm{CH}=\mathrm{CH}_{2}\right), 5.860\left(\mathrm{~m}, 1 \mathrm{H}, \mathrm{OCH}_{2} \mathrm{CH}=\mathrm{CH}_{2}\right), 7.213-7.376(\mathrm{~m}, 10 \mathrm{H}, 2 \mathrm{Ph}) ;{ }^{13} \mathrm{C}, \delta$ 54.8 (OMe), 71.2, 71.3, 72.1, and $73.0\left(2 \mathrm{C}_{6} \mathrm{H}_{5} \mathrm{CH}_{2}, \mathrm{OCH}_{2} \mathrm{CH}=\mathrm{CH}_{2}\right.$, and C-5), 78.3, 79.6, and 80.3 (C-2,3,4), 106.2 (C-1), $117.1\left(\mathrm{OCH}_{2}=\mathrm{CH}_{2}\right), 134.3\left(\mathrm{OCH}_{2} \mathrm{CHCH}=\mathrm{CH}_{2}\right)$, 127.3-128.2, 137.7, and $138.2\left(C_{6} \mathrm{H}_{5} \mathrm{CH}_{2}\right)$.

3-O-Allyl-2,5-di-O-benzyl- $\alpha, \beta$-D-ribofuranose (8). - To a solution of $7(694 \mathrm{mg}$, $1.81 \mathrm{mmol})$ in 1,4-dioxane $(39 \mathrm{~mL})$ was added $1.2 \mathrm{M}$ hydrochloric acid $(9.8 \mathrm{~mL})$, and the mixture was boiled under reflux until hydrolysis was complete $\left(R_{\mathrm{F}} 0.30,95: 5\right.$ dichloromethane-ethyl acetate). The mixture was neutralised with sodium hydrogencarbonate, 
concentrated, diluted with dichloromethane $(40 \mathrm{~mL})$, washed with water $(3 \times 20 \mathrm{~mL})$, dried $\left(\mathrm{MgSO}_{4}\right)$, filtered, and concentrated. The residue was purified by column chromatography (95:5 dichloromethane-ethyl acetate) to yield 8, isolated as a syrup ( $530 \mathrm{mg}$, $79 \% ; \alpha, \beta$-ratio $2: 1),[\alpha]_{\mathrm{D}}+53^{\circ}\left(c 1\right.$, dichloromethane). ${ }^{13} \mathrm{C}$-N.m.r. data $\left(\mathrm{CDCl}_{3}\right): \delta 96.0$ $(\mathrm{C}-1 \alpha), \quad 100.1(\mathrm{C}-1 \beta), \quad 117.1$ and $117.2 \quad\left(\mathrm{OCH}_{2} \mathrm{CH}=\mathrm{CH}_{2}\right), \quad 133.9$ and 134.2 $\left(\mathrm{OCH}_{2} \mathrm{CH}=\mathrm{CH}_{2}\right), 127.3-128.2,137.3$, and $137.7\left(\mathrm{C}_{6} \mathrm{H}_{5} \mathrm{CH}_{2}\right)$.

Anal. Calc. for $\mathrm{C}_{22} \mathrm{H}_{26} \mathrm{O}_{5}: \mathrm{C}, 71.32 ; \mathrm{H}, 7.09$. Found: $\mathrm{C}, 71.53 ; \mathrm{H}, 6.85$.

3-O-Allyl-2,5-di-O-benzyl-D-ribitol (9). - To a solution of 8 (700 $\mathrm{mg}, 1.89 \mathrm{mmol})$ in ethanol $(9.4 \mathrm{~mL})$ was added sodium borohydride $(122 \mathrm{mg})$. After stirring overnight, the reaction was complete $\left(R_{\mathrm{F}} 0.36,7: 3\right.$ dichloromethane-ethyl acetate). The $\mathrm{pH}$ of the mixture was adjusted to 5 with aqueous $96 \%$ acetic acid, and the solution was concentrated, diluted with dichloromethane $(30 \mathrm{~mL})$, washed with $\mathrm{M}$ hydrochloric acid $(2 \times 10$ $\mathrm{mL})$ and water $(3 \times 10 \mathrm{~mL})$, dried $\left(\mathrm{MgSO}_{4}\right)$, filtered, and concentrated. The residue was purified by column chromatography (7:3 dichloromethane ethyl acetate) to yield $\mathbf{9}$, isolated as a syrup $(600 \mathrm{mg}, 85 \%),[\alpha]_{\mathrm{D}}+11^{\circ}(\mathrm{c} 1$, dichloromethane) $)$ N.m.r. data $\left(\mathrm{CDCl}_{3}\right):{ }^{1} \mathrm{H}, 4.069$ and $4.179\left(2 \mathrm{~m}, 2 \mathrm{H}, \mathrm{OCH}_{2} \mathrm{CH}=\mathrm{CH}_{2}\right), 4.505,4.538,4.580$, and 4.620 $\left(4 \mathrm{~d}, 4 \mathrm{H}, 2 \mathrm{PhCH}_{2} \mathrm{O}\right), 5.126$ and $5.204\left(2 \mathrm{~m}, 2 \mathrm{H}, \mathrm{OCH}_{2} \mathrm{CH}=\mathrm{CH}_{2}\right), 5.843(\mathrm{~m}, 1 \mathrm{H}$, $\left.\mathrm{OCH}_{2} \mathrm{CH}=\mathrm{CH}_{2}\right), 7.268-7.352(\mathrm{~m}, 10 \mathrm{H}, 2 \mathrm{Ph}) ;{ }^{13} \mathrm{C}, \delta 60.9(\mathrm{C}-1), 70.5(\mathrm{C}-4), 71.0,71.8$, 72.7, and $73.3\left(2 \mathrm{C}_{6} \mathrm{H}_{5} \mathrm{CH}_{2}, \mathrm{OCH}_{2} \mathrm{CH}=\mathrm{CH}_{2}\right.$, and $\left.\mathrm{C}-5\right), 79.1$ and $79.3(\mathrm{C}-2,3), 117.0$ $\left(\mathrm{OCH}_{2} \mathrm{CH}=\mathrm{CH}_{2}\right), 134.5\left(\mathrm{OCH}_{2} \mathrm{CH}=\mathrm{CH}_{2}\right), 127.7-128.3,137.8$, and $137.9\left(C_{6} \mathrm{H}_{5} \mathrm{CH}_{2}\right)$.

3-O-Allyl-1,2,4,5-tetra-O-benzyl-D-ribitol (10). - To a suspension of sodium hydride ( $100 \mathrm{mg}, 4$ equiv.) in $N, N$-dimethylformamide $(5 \mathrm{~mL})$ was added dropwise at $0^{\circ}$ a solution of 9 ( $382 \mathrm{mg}, 1.03 \mathrm{mmol})$ and benzyl bromide $(0.4 \mathrm{~mL}, 3$ equiv.) in $N, N$-dimethylformamide $(5 \mathrm{~mL})$. The stirring was continued for $2 \mathrm{~h}$ at room temperature when t.l.c. demonstrated that the reaction was complete $\left(R_{\mathrm{F}} 0.20,98: 2\right.$ dichloromethane-ethyl acetate). The excess of sodium hydride was destroyed with methanol, the mixture was poured onto crushed ice and extracted with ether $(3 \times 20 \mathrm{~mL})$, and the combined extracts were dried $\left(\mathrm{MgSO}_{4}\right)$, filtered, and concentrated. The residue was purified by column chromatography ( $98: 2$ dichloromethane-ethyl acetate) to yield 10, isolated as a syrup $(488 \mathrm{mg}, 85 \%)$. N.m.r. data $\left(\mathrm{CDCl}_{3}\right)$ : ${ }^{1} \mathrm{H}, \delta 4.118(\mathrm{~m}, 2 \mathrm{H}$, $\left.\mathrm{OCH}_{2} \mathrm{CH}=\mathrm{CH}_{2}\right), 4.493$ (s), 4.584 (d), and 4.684 (d) $\left(8 \mathrm{H}, 4 \mathrm{PhCH} \mathrm{H}_{2} \mathrm{O}\right), 5.076$ and 5.169 (2 $\mathrm{m}, 2 \mathrm{H}, \mathrm{OCH}_{2} \mathrm{CH}=\mathrm{CH}_{2}$ ), $5.823\left(\mathrm{~m}, 1 \mathrm{H}, \mathrm{OCH}_{2} \mathrm{CH}=\mathrm{CH}_{2}\right.$ ), 7.229-7.344 (m, 20 H, $4 \mathrm{Ph}$ ); ${ }^{13} \mathrm{C}, \delta 70.2,72.3$, and $73.2\left(4 \mathrm{C}_{6} \mathrm{H}_{5} \mathrm{CH}_{2}, \mathrm{C}-1,5\right), 72.2\left(\mathrm{OCH}_{2} \mathrm{CH}=\mathrm{CH}_{2}\right), 78.5(\mathrm{C}-2,4), 78.6$ (C-3), $116.4\left(\mathrm{OCH}_{2} \mathrm{CH}=\mathrm{CH}_{2}\right), 135.0\left(\mathrm{OCH}_{2} \mathrm{CH}=\mathrm{CH}_{2}\right), 127.3-128.2,138.4$, and 138.7 $\left(\mathrm{C}_{6} \mathrm{H}_{5} \mathrm{CH}_{2}\right)$.

Anal. Calc. for $\mathrm{C}_{36} \mathrm{H}_{40} \mathrm{O}_{5}: \mathrm{C}, 78.22 ; \mathrm{H}, 7.31$. Found: C, 78.12; $\mathrm{H}, 7.46$.

I,2,4,5-Tetra-O-benzyl-D-ribitol (11). - A solution of $10(488 \mathrm{mg}, 0.884 \mathrm{mmol})$ in $N, N$-dimethylformamide $(9.1 \mathrm{~mL})$ was heated at $80^{\circ}$, and $\mathrm{KO} \mathrm{O}^{\prime} \mathrm{Bu}(500 \mathrm{mg})$ was added. After $2.5 \mathrm{~h}$, the isomerisation was complete $\left(R_{\mathrm{F}} 0.74,40: 1\right.$ toluene-acetone). The mixture was cooled, dichloromethane $(40 \mathrm{~mL})$ was added, and the organic phase was washed with water $(3 \times 20 \mathrm{~mL})$, dried $\left(\mathrm{MgSO}_{4}\right)$, filtered, and concentrated. The residue was dissolved in 9:1 acetone- $0.1 \mathrm{M}$ hydrochloric acid $(36 \mathrm{~mL})$ and boiled under reflux for 45 min when t.l.c. $\left(R_{F} 0.37,20: 1\right.$ toluene-acetone) showed that the reaction was 
complete. The mixture was neutralised with aqueous $25 \%$ ammonia, concentrated, diluted with dichloromethane $(40 \mathrm{~mL})$, washed with water $(3 \times 20 \mathrm{~mL})$, dried $\left(\mathrm{MgSO}_{4}\right)$, filtered, and concentrated. The residue was purified by column chromatography (20:1 toluene-acetone) to yield 11 , isolated as a syrup (320 mg, $71 \%) .{ }^{13} \mathrm{C}-\mathrm{N} . \mathrm{m} . \mathrm{r}$. data $\left(\mathrm{CDCl}_{3}\right): \delta 70.0,71.9$, and $73.3\left(4 \mathrm{C}_{6} \mathrm{H}_{5} \mathrm{CH}_{2}, \mathrm{C}-1,5\right), 71.5(\mathrm{C}-3), 77.9(\mathrm{C}-2,4), 127.4$ 128.2, 138.0 and $138.2\left(C_{6} \mathrm{H}_{5} \mathrm{CH}_{2}\right)$.

Anal. Calc. for $\mathrm{C}_{33} \mathrm{H}_{36} \mathrm{O}_{5}: \mathrm{C}, 77.30 ; \mathrm{H}, 7.09$. Found: C, 77.11; $\mathrm{H}, 7.23$.

Benzyl 3-O-(3-O-allyl-2,4,6-tri-O-benzyl- $\alpha$-D-glucopyranosyl)-2,4-di-O-benzyl$\alpha$-L-rhamnopyranoside (13). - A solution of ethyl 3- $O$-allyl-2,4,6-tri- $O$-benzyl-1-thio$\beta$-D-glucopyranoside ${ }^{1}(3 ; 6.74 \mathrm{~g}, 12.63 \mathrm{mmol})$ and benzyl 2,4 -di- $O$-benzyl- $\alpha$-L-rhamnopyranoside $^{2}(4 ; 2.58 \mathrm{~g}, 5.92 \mathrm{mmol})$ in dry ether $(50 \mathrm{~mL})$ containing molecular sieves $4 \AA$ $(12.5 \mathrm{~g})$ was stirred for $2 \mathrm{~h}$ in the dark. Methyl triflate $(1.30 \mathrm{~mL}, 11.85 \mathrm{mmol})$ was added and the mixture was stirred overnight when t.l.c. showed that the reaction was not complete. More methyl triflate $(0.62 \mathrm{~mL}, 5.93 \mathrm{mmol})$ was added and, after $4 \mathrm{~h}$, t.l.c. showed the reaction to be complete $\left[R_{\mathrm{F}} 0.33,65: 35\right.$ light petroleum (b.p. $40-60^{\circ}$ ) ether]. Triethylamine $(5.20 \mathrm{~mL})$ was added, the mixture was filtered through Celite and diluted with dichloromethane $(75 \mathrm{~mL}$ ), and the organic phase was washed with aqueous saturated sodium hydrogencarbonate $(2 \times 75 \mathrm{~mL})$ and water $(3 \times 75 \mathrm{~mL})$, dried $\left(\mathrm{MgSO}_{4}\right)$, filtered, and concentrated. The residue was purified by column chromatography [65:35 light petroleum (b.p. $40-60^{\circ}$ )-ether] to yield 13 , isolated as a syrup $(3.27 \mathrm{~g}$, $61 \%),[\alpha]_{D}+22^{\circ}$ (c 1, chloroform). N.m.r. data $\left(\mathrm{CDCl}_{3}\right):{ }^{1} \mathrm{H}, \delta 1.337\left(\mathrm{~d}, 3 \mathrm{H}, J_{5,6} 6.1 \mathrm{~Hz}\right.$, $\mathrm{H}-6,6,6), 3.411$ (dd, $1 \mathrm{H}, J_{6 \mathrm{a}^{\prime}, 6 \mathrm{~b}^{\prime}} 10.7, J_{5^{\prime}, 6 \mathrm{a}^{\prime}} 1.9 \mathrm{~Hz}, \mathrm{H}-6 \mathrm{a}^{\prime}$ ), 3.539 (dd, $1 \mathrm{H}, J_{5^{\prime}, 6 \mathrm{~b}^{\prime}} 2.8 \mathrm{~Hz}$, H-6b'), 3.584 (dd, $1 \mathrm{H}, J_{1^{\prime}, 2^{\prime}} 3.5, J_{2^{\prime}, 3^{\prime}} 9.7 \mathrm{~Hz}, \mathrm{H}-2^{\prime}$ ), 3.884 (dd, $1 \mathrm{H}, J_{2,3} 2.6 \mathrm{~Hz}, \mathrm{H}-2$ ), 3.990 (t, $\left.1 \mathrm{H}, J_{3^{\prime}, 4^{\prime}} 9.7 \mathrm{~Hz}, \mathrm{H}-3^{\prime}\right), 4.142\left(\mathrm{dd}, 1 \mathrm{H}, J_{3,4} 9.7 \mathrm{~Hz}, \mathrm{H}-3\right), 4.339$ and $4.402(2 \mathrm{~m}, 2 \mathrm{H}$, $\left.\mathrm{OCH}_{2} \mathrm{CH}=\mathrm{CH}_{2}\right), 4.284,4.394,4.432,4.540,4.547,4.556,4.621,4.696,4.744,4.811$, 4.824, and 4.878 (12 d, $\left.12 \mathrm{H}, 6 \mathrm{PhCH}_{2} \mathrm{O}\right), 4.777\left(\mathrm{~d}, 1 \mathrm{H}, J_{1,2} 2.0 \mathrm{~Hz}, \mathrm{H}-1\right), 5.120$ and 5.261 (2 m, $\left.2 \mathrm{H}, \mathrm{OCH}_{2} \mathrm{CH}=\mathrm{CH}_{2}\right), 5.133\left(\mathrm{~d}, 1 \mathrm{H}, \mathrm{H}-1^{\prime}\right), 5.973\left(\mathrm{~m}, 1 \mathrm{H}, \mathrm{OCH}_{2} \mathrm{CH}=\mathrm{CH}_{2}\right)$, $7.060-7.342(\mathrm{~m}, 30 \mathrm{H}, 6 \mathrm{Ph}) ;{ }^{13} \mathrm{C}, \delta 17.9(\mathrm{C}-6), 68.0,68.6,73.1(2 \mathrm{C}), 73.2,74.1,74.8$, and $75.4\left(\mathrm{C}-6,6 \mathrm{C}_{6} \mathrm{H}_{5} \mathrm{CH}_{2}\right.$, and $\left.\mathrm{OCH}_{2} \mathrm{CH}=\mathrm{CH}_{2}\right), 68.3,70.2,75.1,76.1,77.7,79.3,80.0$, and $81.8\left(\mathrm{C}-2,3,4,5,2^{\prime}, 3^{\prime}, 4^{\prime}, 5^{\prime}\right), 95.0$ and $97.2\left(\mathrm{C}-1,1^{\prime}\right), 116.3\left(\mathrm{OCH}_{2} \mathrm{CH}=\mathrm{CH}_{2}\right), 127.3-128.3$ and $137.9-138.5\left(\mathrm{C}_{6} \mathrm{H}_{5} \mathrm{CH}_{2}\right), 135.2\left(\mathrm{OCH}_{2} \mathrm{CH}=\mathrm{CH}_{2}\right)$.

Anal. Calc. for $\mathrm{C}_{58} \mathrm{H}_{64} \mathrm{O}_{11}: \mathrm{C}, 75.46 ; \mathrm{H}, 6.90$. Found: C, 75.45; H, 7.04.

Benzyl 2,4-di-O-benzyl-3-O-(2,4,6-tri-O-benzyl- $\alpha$-D-glucopyranosyl)- $\alpha$-L-rhamnopyranoside (14). - A solution of $13(1.20 \mathrm{~g}, 1.33 \mathrm{mmol})$ in $N, N$-dimethylformamide (13 $\mathrm{mL}$ ) was heated at $90^{\circ}$ and $\mathrm{KO} \mathrm{O}^{\mathrm{B}} \mathrm{Bu}(700 \mathrm{mg})$ was added. After $45 \mathrm{~min}$, the reaction was complete $\left(R_{\mathrm{F}} 0.60,25: 1\right.$ toluene-acetone). The mixture was cooled, dichloromethane $(50 \mathrm{~mL})$ was added, and the organic phase washed with water $(13 \mathrm{~mL})$, dried $\left(\mathrm{MgSO}_{4}\right)$, filtered, and concentrated. A solution of the residue in acetone $(12 \mathrm{~mL})$ and $0.1 \mathrm{M}$ hydrochloric acid (1.3 mL) was boiled under reflux for $45 \mathrm{~min}$ when t.l.c. $\left(R_{\mathrm{F}} 0.22,25: 1\right.$ toluene-acetone) showed that the reaction was complete. The mixture was neutralised with aqueous $25 \%$ ammonia, diluted with dichloromethane $(25 \mathrm{~mL})$, washed with water $(3 \times 25 \mathrm{~mL})$, dried $\left(\mathrm{MgSO}_{4}\right)$, filtered, and concentrated. The residue was purified by column chromatography (95:5 toluene-acetone) to yield 14, isolated as a syrup $(1.07 \mathrm{~g}$, 
$93 \%),[\alpha]_{\mathrm{D}}+30^{\circ}\left(c 1\right.$, chloroform). N.m.r. data $\left(\mathrm{CDCl}_{3}\right):{ }^{1} \mathrm{H}, \delta 1.328\left(\mathrm{~d}, 3 \mathrm{H}, J_{5,6} 6.2 \mathrm{~Hz}\right.$, H-6,6,6), 3.476 (dd, $1 \mathrm{H}, J_{6 \mathrm{a}^{\prime}, 6 \mathrm{~b}^{\prime}} 10.6, J_{5^{\prime}, 6 \mathrm{a}^{\prime}} 2.2 \mathrm{~Hz}, \mathrm{H}-6 \mathrm{a}^{\prime}$ ), 3.488 (dd, $1 \mathrm{H}, J_{1^{\prime}, 2^{\prime}} 3.5, J_{2^{\prime}, 3^{\prime}} 9.5$ Hz, H-2'), 3.555 (dd, $\left.1 \mathrm{H}, J_{5^{\prime}, 6 \mathrm{~b}^{\prime}} 3.1 \mathrm{~Hz}, \mathrm{H}-6 \mathrm{~b}^{\prime}\right), 3.745$ (m, $\left.1 \mathrm{H}, \mathrm{H}-5\right), 3.905$ (t, $1 \mathrm{H}, J_{2,3} 2.6$ $\mathrm{Hz}, \mathrm{H}-2), 4.018$ (m, $\left.1 \mathrm{H}, \mathrm{H}-5^{\prime}\right), 4.156$ (dd, $\left.1 \mathrm{H}, J_{3,4} 9.0 \mathrm{~Hz}, \mathrm{H}-3\right), 4.213$ (t, $1 \mathrm{H}, J_{3^{\prime}, 4^{\prime}} 9.5 \mathrm{~Hz}$, H-3'), 4.321, 4.423, 4.481, 4.538, 4.566, 4.581, 4.644, 4.725, 4.769, 4.791, 4.824, and 4.859 (12 d, $\left.12 \mathrm{H}, 6 \mathrm{PhCH}_{2} \mathrm{O}\right), 4.843$ (bs, $\left.1 \mathrm{H}, \mathrm{H}-1\right), 5.227$ (d, $\left.1 \mathrm{H}, \mathrm{H}-1^{\prime}\right), 7.103-7.355$ (m, $30 \mathrm{H}, 6 \mathrm{Ph}) ;{ }^{13} \mathrm{C}, \delta 17.7(\mathrm{C}-6), 67.9,68.5,72.4,72.7,73.0,74.2$, and $75.1\left(\mathrm{C}-6^{\prime}\right.$ and 6 $\left.\mathrm{C}_{6} \mathrm{H}_{5} \mathrm{CH}_{2}\right), 68.1,69.6,73.6,74.9,75.4,77.5,78.8$, and $79.8\left(\mathrm{C}-2,3,4,5,2^{\prime}, 3^{\prime}, 4^{\prime}, 5^{\prime}\right), 93.8$ and $96.8\left(\mathrm{C}-1,1^{\prime}\right), 127.4-128.1$, and $137.5-138.4\left(C_{6} \mathrm{H}_{5} \mathrm{CH}_{2}\right)$.

Anal. Calc. for $\mathrm{C}_{54} \mathrm{H}_{58} \mathrm{O}_{10}: \mathrm{C}, 74.81 ; \mathrm{H}, 6.74$. Found: $\mathrm{C}, 74.34 ; \mathrm{H}, 6.83$.

O- $\alpha$-D-Glucopyranosyl- $(1 \rightarrow 3)$ - $\alpha, \beta$-L-rhamnopyranose (15). - A solution of 14 (245 $\mathrm{mg}, 0.284 \mathrm{mmol}$ ) in ethanol ( $20 \mathrm{~mL}$ ) was hydrogenolysed in the presence of $10 \%$ Pd-C (700 mg) at $4 \mathrm{~atm}$. overnight, filtered through Celite, and concentrated to yield 15 , isolated as a syrup $(74 \mathrm{mg}, 80 \% ; \alpha, \beta$-ratio $2: 1),[\alpha]_{\mathrm{D}}+38^{\circ}(c 1$, water $)$. N.m.r. data $\left(\mathrm{D}_{2} \mathrm{O}\right)$ : ${ }^{1} \mathrm{H}, \delta 1.294$ and $1.311\left(2 \mathrm{~d}\right.$, together $\left.3 \mathrm{H}, J_{5,6} 6.3 \mathrm{~Hz}, \mathrm{H}-6,6,6\right), 4.867(\mathrm{~s})$ and $5.154\left(\mathrm{~d}, J_{1,2}\right.$ $2.0 \mathrm{~Hz}$ ) (together $1 \mathrm{H}, \mathrm{H}-1 \alpha \beta), 5.079$ and $5.106\left(2 \mathrm{~d}\right.$, together $\left.1 \mathrm{H}, J_{1^{\prime}, 2^{\prime}} 3.8 \mathrm{~Hz}, \mathrm{H}-1^{\prime}\right) ;{ }^{13} \mathrm{C}$, $\delta 18.2(\mathrm{C}-6), 61.5\left(\mathrm{C}-6^{\prime}\right), 94.7$ and $94.9(\mathrm{C}-1), 96.5$ and $96.8\left(\mathrm{C}-1^{\prime}\right)$.

Anal. Calc. for $\mathrm{C}_{12} \mathrm{H}_{22} \mathrm{O}_{10} \cdot \mathrm{H}_{2} \mathrm{O}: \mathrm{C}, 41.85 ; \mathrm{H}, 7.04$. Found: C, 42.05; H, 7.08.

Benzyl 2,4-di-O-benzyl-3-O-(2,4,6-tri-O-benzyl-3-O-(2,3,4,6-tetra-O-benzyl- $\alpha$ D-galactopyranosyl)- $\alpha$-D-glucopyranosyl]- $\alpha$-L-rhamnopyranoside (16). - A mixture of 14 (479 mg, $0.553 \mathrm{mmol}), 2$ (650 mg, $1.11 \mathrm{mmol}$ ), and molecular sieves $4 \AA$ ( $3 \mathrm{~g}$ ) in ether $(15 \mathrm{~mL})$ was stirred for $2 \mathrm{~h}$ in the dark at room temperature. Methyl triflate $(120 \mu \mathrm{L}, 1.11$ mmol) was added, and the mixture was stirred for $2 \mathrm{~h}$, when t.l.c. showed that the reaction was not complete. More methyl triflate $(60 \mu \mathrm{L}, 0.553 \mathrm{mmol})$ was added and, after $1 \mathrm{~h}$, the reaction was complete $\left(R_{\mathrm{F}} 0.52,95: 5\right.$ toluene-acetone). Triethylamine (650 $\mu \mathrm{L})$ was added, and the mixture was filtered through Celite, diluted with dichloromethane $(25 \mathrm{~mL})$, washed with aqueous saturated sodium hydrogencarbonate $(2 \times 25$ $\mathrm{mL})$ and water $(3 \times 25 \mathrm{~mL})$, dried $\left(\mathrm{MgSO}_{4}\right)$, filtered, and concentrated. The residue was purified by column chromatography (98.5:1.5 toluene-acetone) to yield 16, isolated as a syrup $(440 \mathrm{mg}, 57 \%),[\alpha]_{\mathrm{D}}+38^{\circ}\left(\mathrm{c} 1\right.$, chloroform). N.m.r. data $\left(\mathrm{CDCl}_{3}\right):{ }^{1} \mathrm{H}, \delta 1.320(\mathrm{~d}, 3$ $\left.\mathrm{H}, J_{5,6} 6.2 \mathrm{~Hz}, \mathrm{H}-6,6,6\right), 4.868\left(\mathrm{~d}, 1 \mathrm{H}, J_{1,2} 2.0 \mathrm{~Hz}, \mathrm{H}-1\right), 5.220\left(\mathrm{~d}, 1 \mathrm{H}, J_{1^{\prime}, 2^{\prime}} 3.4 \mathrm{~Hz}, \mathrm{H}-1^{\prime}\right)$, $5.626\left(\mathrm{~d}, 1 \mathrm{H}, J_{1^{\prime \prime}, 2^{\prime \prime}} 3.4 \mathrm{~Hz}, \mathrm{H}-1^{\prime \prime}\right), 7.056-7.311(\mathrm{~m}, 50 \mathrm{H}, 10 \mathrm{Ph}) ;{ }^{13} \mathrm{C}, \delta 17.8(\mathrm{C}-6), 93.5$, 96.9, and $97.4\left(\mathrm{C}-1,1^{\prime}, 1^{\prime \prime}\right), 126.7-128.3$ and 137.3-138.6 $\left(C_{6} \mathrm{H}_{5} \mathrm{CH}_{2}\right)$.

Anal. Calc. for $\mathrm{C}_{89} \mathrm{H}_{92} \mathrm{O}_{15}: \mathrm{C}, 76.26 ; \mathrm{H}, 6.61$. Found: C, 76.28; $\mathrm{H}, 6.56$.

$\mathrm{O}-\alpha$-D-Galactopyranosyl-( $1 \rightarrow 3)$-O- $\alpha$-D-glucopyranosyl- $(1 \rightarrow 3)-\alpha, \beta$-L-rhamnopyranose (17). - A solution of $16(452 \mathrm{mg}, 0.326 \mathrm{mmol})$ in ethanol $(20 \mathrm{~mL})$ was hydrogenolysed in the presence of $10 \% \mathrm{Pd}-\mathrm{C}(700 \mathrm{mg})$ at $4 \mathrm{~atm}$. overnight, filtered through Celite, and concentrated to yield 17 , isolated as a syrup $(142 \mathrm{mg}, 89 \% ; \alpha, \beta$-ratio $7: 3),[\alpha]_{\mathrm{D}}+1^{\circ}(c$ 1, water). N.m.r. data $\left(\mathrm{D}_{2} \mathrm{O}\right)$ : ${ }^{1} \mathrm{H}, \delta 1.297$ and $1.313\left(2 \mathrm{~d}\right.$, together $3 \mathrm{H}, J_{5,6} 6.3 \mathrm{~Hz}$, $\mathrm{H}-6,6,6), 4.866(\mathrm{~s})$ and $5.154\left(\mathrm{~d}, J_{1,2} 1.7 \mathrm{~Hz}\right.$ ) (together $\left.1 \mathrm{H}, \mathrm{H}-1 \alpha \beta\right), 5.101$ and $5.129(2 \mathrm{~d}$, together $\left.1 \mathrm{H}, J_{1^{\prime}, 2^{\prime}} 3.8 \mathrm{~Hz}, \mathrm{H}-1^{\prime}\right), 5.396\left(\mathrm{~d}, 1 \mathrm{H}, J_{1^{\prime \prime}, 2^{*}} 3.7 \mathrm{~Hz}, \mathrm{H}-1^{\prime \prime}\right) ;{ }^{13} \mathrm{C}, \delta 18.3(\mathrm{C}-6), 61.4$ and $62.2\left(\mathrm{C}-6^{\prime}, 6^{\prime \prime}\right), 94.7$ and $94.9(\mathrm{C}-1), 96.5$ and $96.8\left(\mathrm{C}-1^{\prime}\right), 100.6\left(\mathrm{C}-1^{\prime \prime}\right)$. 
Anal. Calc. for $\mathrm{C}_{18} \mathrm{H}_{32} \mathrm{O}_{15} \cdot 2 \mathrm{H}_{2} \mathrm{O}: \mathrm{C}, 41.21 ; \mathrm{H}, 6.93$. Found: C, 41.32; H, 6.98.

2,4-Di-O-acetyl-3-O-[2,4,6-tri-O-acetyl-3-O-(2,3,4,6-tetra-O-acetyl- $\alpha$-D-galactopyranosyl)- $\alpha$-D-glucopyranosyl]- $\alpha$-L-rhamnopyranose (19). - To a solution of 17 (615 $\mathrm{mg}, 1.26 \mathrm{mmol})$ in acetic anhydride pyridine $(1: 1,20 \mathrm{~mL})$ was added a catalytic amount of 4-dimethylaminopyridine. After stirring overnight at $50^{\circ}$, the reaction was complete $\left(R_{\mathrm{F}} 0.34,98: 2\right.$ dichloromethane-methanol). The mixture was thrice co-concentrated with toluene $(30 \mathrm{~mL})$, ethanol $(30 \mathrm{~mL})$, and dichloromethane $(30 \mathrm{~mL})$ to yield 18 , isolated quantitatively as a syrup, $[\alpha]_{\mathrm{D}}+97^{\circ}(c 1$, chloroform); $\alpha, \beta$-ratio $1: 1$.

Anal. Calc. for $\mathrm{C}_{38} \mathrm{H}_{52} \mathrm{O}_{25}: \mathrm{C}, 50.21 ; \mathrm{H}, 5.78$. Found: C, 49.83; H, 5.80.

To a solution of $18(1.26 \mathrm{~g}, 1.39 \mathrm{mmol})$ in $N, N$-dimethylformamide $(14 \mathrm{~mL})$ was added hydrazine acetate $(163 \mathrm{mg}, 1.77 \mathrm{mmol})$. The mixture was stirred for $6 \mathrm{~h}$ when the deacetylation was complete $\left(R_{\mathrm{F}} 0.39,6: 4\right.$ dichloromethane-ethyl acetate). Ethyl acetate $(50 \mathrm{~mL})$ was added, and the mixture was diluted with dichloromethane $(50 \mathrm{~mL})$, washed with aqueous $5 \%$ sodium chloride $(3 \times 100 \mathrm{~mL})$, dried $\left(\mathrm{MgSO}_{4}\right)$, filtered, and concentrated. The residue was purified by column chromatography ( $7: 3$ dichloromethaneethyl acetate) to yield 19 , isolated as a syrup $(1.06 \mathrm{~g}, 88 \%),[\alpha]_{\mathrm{D}}+102^{\circ}(c 1$, chloroform $)$. ${ }^{13}$ C-N.m.r. data $\left(\mathrm{CDCl}_{3}\right): \delta 17.2(\mathrm{C}-6), 20.3-20.6\left(\mathrm{CH}_{3} \mathrm{CO}\right), 60.6$ and $61.4\left(\mathrm{C}-6^{\prime}, 6^{\prime \prime}\right), 91.7$ $\left(J_{\mathrm{C}, \mathrm{H}} 170.8 \mathrm{~Hz}\right), 92.9\left(J_{\mathrm{C}, \mathrm{H}} 173.9 \mathrm{~Hz}\right)$, and $95.8\left(J_{\mathrm{C}, \mathrm{H}} 175.9 \mathrm{~Hz}\right)\left(\mathrm{C}-1,1^{\prime}, 1^{\prime \prime}\right), 169.2-170.4$ $\left(\mathrm{CH}_{3} \mathrm{CO}\right)$.

Anal. Calc. for $\mathrm{C}_{36} \mathrm{H}_{50} \mathrm{O}_{24}: \mathrm{C}, 49.87 ; \mathrm{H}, 5.82$. Found: C, 49.41; H, 5.95.

2,4-Di-O-acetyl-3-O-[2,4,6-tri-O-acetyl-3-O-(2,3,4,6-tetra-O-acetyl- $\alpha$-D-galactopyranosyl)- $\alpha$-D-glucopyranosyl]- $\alpha$-L-rhamnopyranosyl trichloroacetimidate (20). - To a solution of $19(155 \mathrm{mg}, 0.179 \mathrm{mmol})$ in dichloromethane $(2.0 \mathrm{~mL})$ and trichloroacetonitrile $(225 \mu \mathrm{L}, 2.24 \mathrm{mmol})$ was added at $-5^{\circ}$ a solution of 1,8-diazabicyclo[5.4.0]undec-7-ene (DBU; $30 \mu \mathrm{L}, 0.20 \mathrm{mmol}$ ) in dichloromethane $(1.0 \mathrm{~mL})$. The mixture was stirred for $1 \mathrm{~h}$ when the reaction was complete $\left(R_{\mathrm{F}} 0.47,9: 1\right.$ dichloromethane-acetone) and, after concentration, the residue was purified by column chromatography $(9: 1$ toluene-acetone) to yield 20 , isolated as a syrup $(146 \mathrm{mg}, 81 \%),[\alpha]_{\mathrm{D}}+33^{\circ}(\mathrm{c} 1$, chloroform). N.m.r. data $\left(\mathrm{CDCl}_{3}\right):{ }^{1} \mathrm{H}, \delta 1.257\left(\mathrm{~d}, 3 \mathrm{H}, J_{5,6} 6.2 \mathrm{~Hz}, \mathrm{H}-6,6,6\right), 1.964,2.052$, $2.060,2.080,2.100,2.105,2.107,2.120$, and 2.194 (9 s, each $3 \mathrm{H}, 9 \mathrm{Ac}$ ), 4.854 (dd, $1 \mathrm{H}$, $\left.J_{1^{\prime}, z^{\prime}} 3.4, J_{2^{\prime}, 3^{\prime}} 10.2 \mathrm{~Hz}, \mathrm{H}-2^{\prime}\right), 5.191\left(\mathrm{~d}, 1 \mathrm{H}, \mathrm{H}-1^{\prime}\right), 5.220$ (dd, $\left.1 \mathrm{H}, \mathrm{H}-3^{\prime \prime}\right), 5.285$ (d, 1 H, $J_{1^{\prime \prime}, z^{\prime \prime}}$ $\left.3.7 \mathrm{~Hz}, \mathrm{H}-1^{\prime \prime}\right), 5.399\left(\mathrm{dd}, 1 \mathrm{H}, J_{4^{\prime \prime}, 5^{\prime \prime}} 0.9, J_{3^{\prime \prime}, 4^{\prime \prime}} 3.4 \mathrm{~Hz}, \mathrm{H}-4^{\prime \prime}\right), 5.438$ (t, $\left.1 \mathrm{H}, \mathrm{H}-2\right), 6.193$ (d, 1 $\left.\mathrm{H}, J_{1,2} 2.7 \mathrm{~Hz}, \mathrm{H}-1\right) ;{ }^{13} \mathrm{C}, \delta 17.1(\mathrm{C}-6), 20.1-20.3\left(\mathrm{CH}_{3} \mathrm{CO}\right), 60.4$ and $61.4\left(\mathrm{C}-6^{\prime}, 6^{\prime \prime}\right), 90.2$ $\left(\mathrm{OC}=\mathrm{NHCCl}_{3}\right), 93.1,94.5$, and $95.6\left(\mathrm{C}-1,1^{\prime}, 1^{\prime \prime}\right), 159.1\left(\mathrm{OC}=\mathrm{NHCCl}_{3}\right), 169.0-170.2$ $\left(\mathrm{CH}_{3} \mathrm{CO}\right)$.

1,2,4,5-Tetra-O-benzyl-3-O-\{2,4-di-O-acetyl-3-O-[2,4,6-tri-O-acetyl-3-O- $(2,3,4$, 6 -tetra-O-acetyl- $\alpha-\mathrm{D}-$ galactopyranosyl)- $\alpha-\mathrm{D}-$ glucopyranosyl]- $\alpha-\mathrm{L}-\mathrm{rhamnopyranosyl}\}-\mathrm{D}-$ ribitol (21). - A suspension of $20(200 \mathrm{mg}, 0.20 \mathrm{mmol}), 11(130 \mathrm{mg}, 0.30 \mathrm{mmol})$, and molecular sieves $4 \AA(2 \mathrm{~g})$ in dichloromethane $(5 \mathrm{~mL})$ was stirred for $2 \mathrm{~h}$ at room temperature. Then, at $-30^{\circ}$, trimethylsilyl triflate $(15.4 \mu \mathrm{L})$ was added and the mixture was stirred for $5 \mathrm{~min}$, when t.l.c. showed the reaction to be complete $\left(R_{\mathrm{F}} 0.46,8: 2\right.$ dichloromethane-ethyl acetate). Pyridine $(1.0 \mathrm{~mL})$ was added, and the mixture was filtered through Celite, diluted with dichloromethane $(50 \mathrm{~mL})$, washed with water $(3 \times$ 
$10 \mathrm{~mL}$ ), dried $\left(\mathrm{MgSO}_{4}\right)$, filtered, and concentrated. The residue was purified by column chromatography (8:2 dichloromethane-ethyl acetate) to yield 21 , isolated as a syrup (242 mg, 90\%), $[\alpha]_{\mathrm{D}}+46^{\circ}$ (c 1, dichloromethane). N.m.r. data $\left(\mathrm{CDCl}_{3}\right):{ }^{1} \mathrm{H}, \delta 0.998(\mathrm{~d}, 3$ $\left.\mathrm{H}, J_{5^{\prime}, 6^{\prime}} 6.2 \mathrm{~Hz}, \mathrm{H}-6^{\prime}, 6^{\prime}, 6^{\prime}\right), 1.954,2.031,2.043,2.048,2.051,2.070,2.073,2.092$, and 2.110 $(9 \mathrm{~s}$, each $3 \mathrm{H}, 9 \mathrm{Ac}), 4.853$ (dd, $\left.1 \mathrm{H}, J_{1^{\prime \prime}, 2^{\prime \prime}} 3.4, J_{2^{*}, 3^{\prime \prime}} 10.1 \mathrm{~Hz}, \mathrm{H}-2^{\prime \prime}\right), 4.990$ (d, $1 \mathrm{H}, J_{1^{\prime}, 2^{\prime}} 1.7$ Hz, H-1'), 5.139 (d, $\left.1 \mathrm{H}, \mathrm{H}-1^{\prime \prime}\right), 5.257$ (d, $\left.1 \mathrm{H}, J_{1^{\prime \prime}, 2^{\prime \prime}} 3.6 \mathrm{~Hz}, \mathrm{H}-1^{\prime \prime \prime}\right), 5.383$ (dd, $1 \mathrm{H}, J_{3^{\prime \prime \prime}, 4^{\prime \prime}} 3.3$ $\left.\mathrm{Hz}, \mathrm{H}-4^{\prime \prime \prime}\right), 7.190-7.321(\mathrm{~m}, 20 \mathrm{H}, 4 \mathrm{Ph}) ;{ }^{13} \mathrm{C}, \delta 17.1\left(\mathrm{C}-6^{\prime}\right), 20.4-20.7\left(\mathrm{CH}_{3} \mathrm{CO}\right), 60.7$ and $61.4\left(\mathrm{C}-6^{\prime \prime}, 6^{\prime \prime \prime}\right), 68.2$ and $69.7(\mathrm{C}-1,5), 71.7$ and $71.9\left(4 \mathrm{C}_{6} \mathrm{H}_{5} \mathrm{CH}_{2}\right), 93.1,95.9$, and 97.4 $\left(\mathrm{C}-1^{\prime}, 1^{\prime \prime}, 1^{\prime \prime \prime}\right), 127.3-128.2$ and 137.9-138.1 $\left(\mathrm{C}_{6} \mathrm{H}_{5} \mathrm{CH}_{2}\right), 169.2-170.5\left(\mathrm{CH}_{3} \mathrm{CO}\right)$.

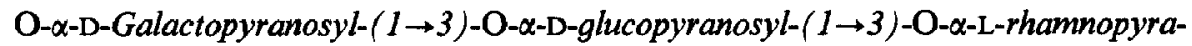
nosyl-( $1 \rightarrow 3)$-D-ribitol (23). - To a solution of $21(200 \mathrm{mg}, 0.15 \mathrm{mmol})$ in dry methanol (4 $\mathrm{mL}$ ) was added sodium methoxide ( $\mathrm{pH} \mathrm{10).} \mathrm{After} \mathrm{stirring} \mathrm{for} 48 \mathrm{~h}$ at room temperature, deacetylation was complete $\left(R_{\mathrm{F}} 0.12,9: 1\right.$ dichloromethane-methanol). The mixture was neutralised with Dowex-50 $\left(\mathrm{H}^{+}\right)$resin, filtered, and concentrated to yield 22 , isolated as a syrup $(145 \mathrm{mg}, 98 \%),[\alpha]_{\mathrm{D}}+55^{\circ}(\mathrm{c} 1$, methanol). A solution of 22 in methanol $(20 \mathrm{~mL})$ was hydrogenolysed overnight in the presence of $10 \% \mathrm{Pd}-\mathrm{C}(700 \mathrm{mg})$ at $4 \mathrm{~atm}$., then filtered through Celite, and concentrated to yield 23 , isolated as a syrup ( $85 \mathrm{mg}, 93 \%)$, $[\alpha]_{\mathrm{D}}+26^{\circ}$ (c 1, water). N.m.r. data $\left(\mathrm{D}_{2} \mathrm{O}\right):{ }^{1} \mathrm{H}, \delta 5.019\left(\mathrm{bs}, 1 \mathrm{H}, \mathrm{H}-1^{\prime}\right), 5.110\left(\mathrm{~d}, 1 \mathrm{H}, J_{1^{\prime \prime}, 2^{*}}\right.$ $3.7 \mathrm{~Hz}, \mathrm{H}-1^{\prime \prime}$ ), 5.396 (d, $\left.1 \mathrm{H}, J_{1^{\prime \prime}, 2^{\prime \prime \prime}} 3.8 \mathrm{~Hz}, \mathrm{H}-1^{\prime \prime \prime}\right)$; ${ }^{13} \mathrm{C}, \delta 18.0\left(\mathrm{C}-6^{\prime}\right), 96.7\left(\mathrm{C}-1^{\prime \prime}\right), 100.5$ (C-1"'), $101.4\left(\mathrm{C}-1^{\prime}\right)$.

Anal. Calc. for $\mathrm{C}_{23} \mathrm{H}_{42} \mathrm{O}_{19} \cdot 3 \mathrm{H}_{2} \mathrm{O}: \mathrm{C}, 40.83 ; \mathrm{H}, 7.15$. Found: C, 41.19; $\mathrm{H}, 7.09$.

1-O-Allyloxycarbonyl-2,3,5-tri-O-benzyl-4-O- $\{2,4-$ di-O-acetyl-3-O-[2,4,6-tri-Oacetyl-3-0-(2,3,4,6-tetra-O-acetyl- $\alpha-\mathrm{D}-$ galactopyranosyl)- $\alpha-\mathrm{D}-$ glucopyranosyl]- $\alpha-\mathrm{L}-$ rhamnopyranosyl \}-D-ribitol (24). - A suspension of 12 (ref. 3) (75 mg, $0.16 \mathrm{mmol}$ ), 20 $(127 \mathrm{mg}, 0.126 \mathrm{mmol})$, and powdered molecular sieves $4 \AA(2 \mathrm{~g})$ in dichloromethane $(4.5$ $\mathrm{mL})$ was stirred for $2 \mathrm{~h}$ under nitrogen at room temperature. Then, at $-30^{\circ}$, trimethylsilyl triflate $(12 \mu \mathrm{L})$ was added, and the mixture was stirred for $5 \mathrm{~min}$, when t.l.c. showed the reaction to be complete $\left(R_{F} 0.48,8: 2\right.$ dichloromethane-ethyl acetate). Pyridine (1.0 $\mathrm{mL})$ was added, and the mixture was filtered through Celite, and co-concentrated thrice each with toluene $(10 \mathrm{~mL})$, ethanol $(10 \mathrm{~mL})$, and dichloromethane $(10 \mathrm{~mL})$. The residue was purified by column chromatography ( $9: 1$ dichloromethane-ethyl acetate) to yield 24 , isolated as a syrup $(150 \mathrm{mg}, 91 \%),[\alpha]_{\mathrm{D}}+29^{\circ}\left(c 1\right.$, chloroform). N.m.r. data $\left(\mathrm{CDCl}_{3}\right)$ : ${ }^{1} \mathrm{H}, \delta 1.035\left(\mathrm{~d}, 3 \mathrm{H}, J_{5^{\prime}, 6^{\prime}} 6.2 \mathrm{~Hz}, \mathrm{H}-6^{\prime}, 6^{\prime}, 6^{\prime}\right), 1.964,2.023,2.049,2.065,2.077,2.085,2.122$, and $2.146(8 \mathrm{~s}, 3,3,6,3,3,3,3$, and $3 \mathrm{H}, 9 \mathrm{Ac}), 4.382,4.425,4.477,4.567,4.643$, and 4.655 (6 d, $6 \mathrm{H}, 3 \mathrm{PhCH}_{2} \mathrm{O}$ ), 4.870 (dd, $\left.1 \mathrm{H}, \mathrm{H}-2^{\prime \prime}\right), 5.121$ (d, $\left.1 \mathrm{H}, J_{1^{\prime}, 2^{\prime}} 1.8 \mathrm{~Hz}, \mathrm{H}-1^{\prime}\right), 5.177$ (d, 1 $\left.\mathrm{H}, J_{1^{\prime \prime}, 2^{\prime \prime}} 3.4 \mathrm{~Hz}, \mathrm{H}-1^{\prime \prime}\right), 5.271$ (d, $\left.1 \mathrm{H}, J_{1^{\prime \prime \prime}, 2^{\prime \prime \prime}} 3.3 \mathrm{~Hz}, \mathrm{H}-1^{\prime \prime \prime}\right), 5.392$ (dd, $1 \mathrm{H}, J_{3^{\prime \prime}, 4^{\prime \prime \prime}} 3.3 \mathrm{~Hz}$, $\mathrm{H}-4^{\prime \prime \prime}$ ), 5.911 (m, $1 \mathrm{H}, \mathrm{OCOOCH}_{2} \mathrm{CH}=\mathrm{CH}_{2}$ ), 7.221-7.337 (m, $\left.15 \mathrm{H}, 3 \mathrm{Ph}\right) ;{ }^{13} \mathrm{C}, \delta 17.2$ $\left(\mathrm{C}-6^{\prime}\right), 20.5-20.8\left(\mathrm{CH}_{3} \mathrm{CO}\right), 60.7$ and $61.5\left(\mathrm{C}-6^{\prime \prime}, 6^{\prime \prime}\right), 93.2,96.0$, and $96.8\left(\mathrm{C}-1^{\prime}, 1^{\prime \prime}, 1^{\prime \prime \prime}\right)$, $118.8\left(\mathrm{OCOOCH}_{2} \mathrm{CH}=\mathrm{CH}_{2}\right), \quad 127.6-128.3$ and $137.5-137.8 \quad\left(C_{6} \mathrm{H}_{5} \mathrm{CH}_{2}\right), \quad 131.5$ $\left(\mathrm{OCOOCH}_{2} \mathrm{CH}=\mathrm{CH}_{2}\right), 154.7\left(\mathrm{OCOOCH}_{2} \mathrm{CH}=\mathrm{CH}_{2}\right), 169.3-170.6\left(\mathrm{CH}_{3} \mathrm{CO}\right)$.

2,3,5-Tri-O-benzyl-4-O-\{2,4-di-O-acetyl-3-O-[2,4,6-tri-O-acetyl-3-O- $(2,3,4,6$ tetra-O-acetyl- $\alpha$-D-galactopyranosyl)- $\alpha$-D-glucopyranosyl $]-\alpha-\mathrm{L}-r h a m n o p y r a n o s y l\}-\mathrm{D}-$ ribitol (25). - To a solution of $24(182 \mathrm{mg}, 0.135 \mathrm{mmol})$ in tetrahydrofuran $(1.7 \mathrm{~mL})$ was 
added palladium tetrakis[triphenylphosphine] $(16 \mathrm{mg}, 14 \mu \mathrm{mol})$, and the mixture was boiled under reflux for 2.5 h. T.l.c. $\left(R_{\mathrm{F}} 0.35,9: 1\right.$ dichloromethane-ethyl acetate $)$ then showed complete conversion into 25 . The mixture was cooled and thrice co-concentrated with 1,4-dioxane $(10 \mathrm{~mL})$. The residue was purified by column chromatography $(9: 1$ dichloromethane-ethyl acetate) to yield 25 , isolated as a syrup $(141 \mathrm{mg}, 82 \%),[\alpha]_{\mathrm{D}}$ $+50^{\circ}$ (c 1, chloroform). N.m.r. data $\left(\mathrm{CDCl}_{3}\right):{ }^{1} \mathrm{H}, \delta 1.057\left(\mathrm{~d}, 3 \mathrm{H}, J_{5^{\prime}, 6^{\prime}} 6.8 \mathrm{~Hz}, \mathrm{H}-6^{\prime}, 6^{\prime}, 6^{\prime}\right)$, $1.962,2.022,2.047,2.064,2.076,2.091,2.119$, and $2.151(8 \mathrm{~s}, 3,3,6,3,3,3,3$, and $3 \mathrm{H}, 9$ Ac), 4.398, 4.444, 4.505, 4.574, 4.596, and 4.679 (6 d, $6 \mathrm{H}, 3 \mathrm{PhCH}_{2} \mathrm{O}$ ), 4.866 (dd, $1 \mathrm{H}$, $J_{1^{\prime \prime}, 2^{\prime \prime}} 3.4, J_{2^{\prime \prime}, 3^{\prime \prime}} 10.2 \mathrm{~Hz}, \mathrm{H}-2^{\prime \prime}$ ), 5.142 (d, $1 \mathrm{H}, J_{1^{\prime}, 2^{\prime}} 1.8 \mathrm{~Hz}, \mathrm{H}-1^{\prime}$ ), 5.192 (d, 1 H, H-1"), 5.279 (d, $\left.1 \mathrm{H}, J_{1^{\prime \prime \prime}, 2^{\prime \prime}} 3.5 \mathrm{~Hz}, \mathrm{H}-1^{\prime \prime \prime}\right), 5.397$ (dd, $\left.1 \mathrm{H}, J_{3^{\prime \prime \prime}, 4^{\prime \prime}} 3.4 \mathrm{~Hz}, \mathrm{H}-4^{\prime \prime \prime}\right), 7.231-7.347$ (m, $15 \mathrm{H}, 3$ $\mathrm{Ph}) ;{ }^{13} \mathrm{C}, \delta 17.3\left(\mathrm{C}-6^{\prime}\right), 20.5-20.7\left(\mathrm{CH}_{3} \mathrm{CO}\right), 60.7$ and $61.5\left(\mathrm{C}-6 " 6^{\prime \prime}, 6^{\prime \prime}\right), 61.0$ (C-1), 70.1, 72.1, 73.2, and 73.5 (C-5 and $\left.3 \mathrm{C}_{6} \mathrm{H}_{5} \mathrm{CH}_{2}\right), 93.0,95.9$, and $96.9\left(\mathrm{C}-1^{\prime}, 1^{\prime \prime}, 1^{\prime \prime \prime}\right), 127.6-128.3$ and 137.6-137.8 $\left(\mathrm{C}_{6} \mathrm{H}_{5} \mathrm{CH}_{2}\right), 169.2-170.5\left(\mathrm{CH}_{3} \mathrm{CO}\right)$.

O- $\alpha$-D-Galactopyranosyl- $(1 \rightarrow 3)-\mathrm{O}-\alpha-\mathrm{D}-$ glucopyranosyl- $(1 \rightarrow 3)-\mathrm{O}-\alpha-\mathrm{L}-$ rhamnopyranosyl-(1 $\rightarrow 4)$-D-ribitol (27). - To a solution of $25(103 \mathrm{mg}, 81 \mu \mathrm{mol})$ in dry methanol $(1.0 \mathrm{~mL})$ was added sodium methoxide $(\mathrm{pH} 10)$. After stirring overnight at room temperature, deacetylation was complete $\left(R_{\mathrm{F}} 0.73,1: 1\right.$ dichloromethane-methanol). The mixture was neutralised with Dowex- $50\left(\mathrm{H}^{+}\right)$resin, filtered, and concentrated to yield 26, isolated quantitatively as a syrup. A solution of $26(45.3 \mathrm{mg}, 50.8 \mu \mathrm{mol})$ in ethanol $(10.0 \mathrm{~mL})$ was hydrogenolysed in the presence of $10 \% \mathrm{Pd}-\mathrm{C}(350 \mathrm{mg})$ at $4 \mathrm{~atm}$. overnight, filtered through Celite, and concentrated to yield 27 , isolated as a syrup (28 $\mathrm{mg}, 90 \%),[\alpha]_{\mathrm{D}}-25^{\circ}$ (c 1, water). N.m.r. data $\left(\mathrm{D}_{2} \mathrm{O}\right):{ }^{1} \mathrm{H}, \delta 5.081$ (bs, $\left.1 \mathrm{H}, \mathrm{H}-1^{\prime}\right), 5.124$ (d, $\left.1 \mathrm{H}, J_{1^{\prime \prime}, 2^{\prime \prime}} 3.7 \mathrm{~Hz}, \mathrm{H}-1^{\prime \prime}\right), 5.390\left(\mathrm{~d}, 1 \mathrm{H}, J_{1^{\prime \prime}, 2^{m}} 3.6 \mathrm{~Hz}, \mathrm{H}-1^{\prime \prime \prime}\right) ;{ }^{13} \mathrm{C}, \delta 18.0\left(\mathrm{C}-6^{\prime}\right), 96.8\left(\mathrm{C}-1^{\prime \prime}\right)$, $100.6\left(\mathrm{C}-1^{\prime \prime \prime}\right), 101.0\left(\mathrm{C}-1^{\prime}\right)$.

Anal. Calc. for $\mathrm{C}_{23} \mathrm{H}_{42} \mathrm{O}_{19} \cdot 3 \mathrm{H}_{2} \mathrm{O}: \mathrm{C}, 40.83 ; \mathrm{H}, 7.15$. Found: C, 41.23; H, 7.24.

\section{ACKNOWLEDGMENTS}

This investigation was supported by the Netherlands Foundation for Chemical Research (SON) with financial aid from the Netherlands Organization for Scientific Research (NWO), by the Institute of Molecular Biology and Medical Biotechnology (IMB, Utrecht University), and by the Netherlands Innovation Directed Programme for Biotechnology (IOP-b).

\section{REFERENCES}

1 T. M. Slaghek, M. J. van Vliet, A. A. M. Maas, J. P. Kamerling, and J. F. G. Vliegenthart, Carbohydr. Res., 195 (1989) 75-86.

2 V. Poszgay, Carbohydr. Res., 69 (1979) $284-286$.

3 P. Boullanger, P. Chatelard, G. Descotes, M. Kloosterman, and J. H. van Boom, J. Carbohydr. Chem., 5 (1986) 541-559.

4 M. Cerny, J. Stanek, and J. Pacak, Monatsh. Chem., 94 (1963) 290-294.

5 A. F. Bochkov, V. M. Dashunin, and N. K. Kochetkov, Izv. Akad. Nauk SSSR., Ser. Khim., 3 (1975) 632-638. 
6 R. U. Lemieux, Can. J. Chem., 29 (1951) 10791091.

7 G. M. Tener and H. G. Khorana, J. Am. Chem. Soc., 79 (1957) 437-441.

8 A. Lipták, P. Fügedi, and P. Nánási, Carbohydr. Res., 65 (1978) 209-217.

9 P. Fügedi, P. J. Garcgg, H. Lönn, and T. Norberg, Glycoconj. J., 4 (1987) 97108.

10 G. Excoffier, D. Gagnaire, and J.-P. Utille, Carbohydr. Res., 39 (1975) 368-375.

11 S. Sato, Y. Ito, T. Nukada, Y. Nakahara, and T. Ogawa, Carbohydr. Res., 167 (1987) 197-210.

12 J. E. G. van Dam, J. Breg, R. Komen, J. P. Kamerling, and J. F. G. Vliegenthart, Carbohydr. Res., 187 (1989) 267-286. 